\title{
I Jornadas Patagónicas de Ciencias del Ambiente y Recursos Naturales
}

\author{
Zulma LIZARRALDE \\ icasur@uarg.unpa.edu.ar
}

\section{Directora ICASUR, UARG, UNPA}

Las "I Jornadas Patagónicas de Ciencias del Ambiente y Recursos Naturales", se realizaron en el Campus Universitario de la Unidad Académica Río Gallegos de la Universidad Nacional de la Patagonia Austral, el 10 de octubre de 2018. Fue organizado por el Instituto de Ciencias del Ambiente, Sustentabilidad y Recursos Naturales (ICASUR), y las Secretarias de Extensión e Investigación y Posgrado de la Unidad Académica Río Gallegos.

El encuentro tuvo como objetivo generar un espacio de debate e intercambio de experiencias y conocimiento entre los equipos de investigación del ICASUR-UNPA, y de Universidades e Instituciones Patagónicas de investigación. Asimismo, permitió difundir en la comunidad los trabajos desarrollados en el ámbito del Instituto.

El Consejo Directivo del Instituto ha establecido que la Jornadas se organizarán cada dos años en las diferentes sedes del ICASUR (Unidades Académicas Río Turbio, San Julián, Caleta Olivia y Río Gallegos de la UNPA), con el fin de favorecer el intercambio entre los investigadores, becarios y alumnos, así como también divulgar en la sociedad los resultados de sus investigaciones.

En las Primeras Jornadas, se presentaron trabajos de investigadores, estudiantes y becarios de grado y posgrado, relacionados con los ejes temáticos: 1) Ambiente y recursos naturales, 2) Diversidad biológica, 3) Manejo y conservación de recursos naturales y culturales, 4) Producción de recursos naturales, 5) Ordenamiento territorial y planificación urbana, 6) Turismo sustentable.

La conferencia inaugural titulada "Los cambios climáticos globales durante el Cuaternario y su impacto en la Patagonia" estuvo a cargo del Dr. Jorge Rabassa del Centro Austral de Investigaciones Científicas (CADIC-CONICET, Ushuaia).

Se presentaron un total de 35 ponencias y tres posters de becarios de grado y posgrado. El libro de resúmenes se encuentra disponible en https://www.uarg.unpa.edu.ar 